Review article

\title{
Bedaquiline - The first ATP synthase inhibitor against multi drug resistant tuberculosis
}

\author{
Mageshwaran Lakshmanan*, Alphienes Stanley Xavier \\ Department of Pharmacology, Jawaharlal Institute of Postgraduate Medical Education and Research, Pondicherry 605006, India
}

\section{A R T I C L E I N F O}

\section{Article history:}

Received 6 November 2013

Accepted 3 December 2013

Available online 30 December 2013

\section{Keywords:}

Bedaquiline

MDR-TB

XDR-TB

ATP synthase inhibitor

Tuberculosis

\begin{abstract}
A B S T R A C T
Increasing incidence of MDR-TB, long duration of treatment and co-infection with HIV are the significant problems in achieving the eradication of tuberculosis. Bedaquiline is an anti-tuberculosis drug with unique mechanism of action. It selectively inhibits the mycobacterial energy metabolism i.e. ATP synthesis and found to be effective against all states of Mycobacterium tuberculosis like active, dormant, replicating, non-replicating, intracellular and extracellular. Preclinical studies have shown the efficacy of bedaquiline in terms of reduction in bacterial load and treatment duration. Phase II clinical studies have established the safety, tolerability and earlier sputum conversion time in patients with MDR-TB. In 2012 FDA approved bedaquiline for treatment of MDR-TB and XDR-TB.
\end{abstract}

Copyright (c) 2013, InPharm Association, Published by Reed Elsevier India Pvt. Ltd. All rights reserved.

\section{Introduction}

Tuberculosis (TB), caused predominantly by Mycobacterium tuberculosis (M. tb) and other closely related species, is a disease of antiquity with extraordinary endurance and versatile clinical features. With an estimated incidence of 8.7 million cases per year, approximately it causes 1.4 million deaths world-wide annually. ${ }^{1}$ Even though TB cannot be eradicated by 2050 based on current incidence rate, many governmental and non-governmental services have taken enormous steps to combat it. However for the past two decades, emergence of resistance against rifampicin ( $R$ ) and isoniazid $(\mathrm{H})$ called multidrug resistant TB (MDR-TB), resistance against $\mathrm{R}, \mathrm{H}$, a fluoroquinolone (FQ) and a second line injectables called as extensively drug resistant TB (XDR-TB) and recently resistance against all drugs commonly used in drug susceptibility testing called totally drug resistant TB (TDR-TB) demands the improved and better tussle against TB. In 2011, around 3,10,000 cases of MDRTB were detected globally amongst the notified pulmonary TB patients with high prevalence in India, China and Russian federations. It has also been estimated that about $9 \%$ of notified MDR-TB cases includes XDR-TB. ${ }^{2}$

The World Health Organization currently recommends a combination of an aminoglycoside (Amikacin-AMK), ethionamide (ETH), a fluoroquinolone (Moxifloxacin-MOXF) and pyrazinamide $(\mathrm{Z})$ for MDR-TB for minimum period of 20 months. Nevertheless the existing

\footnotetext{
* Corresponding author. Tel.: +919788799896 (mobile).

E-mail address: waranmagesh87@gmail.com (M. Lakshmanan).
}

treatment regimens for management of MDR-TB and XDR-TB are unsatisfactory and take considerably long duration to treat, which can result in emergence of drug specific side effects, significant adverse drug reactions and untoward drug-to-drug interactions.

Along with HIV infection, the incidence of TB worldwide has become more threatening. Globally, amongst 8.7 million TB patients, $13 \%$ are co-infected with HIV. Moreover, incidence of MDR and XDR-TB is higher among HIV co-infected TB patients. (1) In all these circumstances, significant drug-to-drug interactions can occur when the anti-TB drugs are administered along with anti-HIV drugs. These interactions may lead to failure in the efficacy of antiTB drugs or increased toxicity of anti-HIV drugs or vice-versa.

In March 2010 an initiative called Critical path to New TB regimens (CPTR) has been established by critical path institute and TB alliance. This CPTR initiative is a motivation for the development of new drug combinations and incorporation of newer drugs into existing regimen to deliver the best against $\mathrm{TB}^{3}{ }^{3}$

On considering all these facts, we need a drug, which can be principally used to treat MDR-TB and XDR-TB, significantly decrease the duration of treatment and thereby prevents the emergence of MDR-TB and XDR-TB with less drug interaction properties. This may be achieved by developing a drug with novel mechanism of action, which can be used in combinations.

Bedaquiline (TMC207, R207910 or Compound J) targets against Mycobacterial ATP synthase complex. Bedaquiline was recently approved by FDA in 2012 for treatment of MDR-TB at a dose of $400 \mathrm{mg}$ once daily for 2 weeks followed by $200 \mathrm{mg} 3$ times per week for next 22 weeks. 


\section{Mechanism of action}

Bedaquiline, chemically belongs to diarylquinoline (DAR) compound, is closely related to fluoroquinolones as well as chloroquine but with different side-chain moiety. Amongst all anti-TB drugs approved, bedaquiline is the only drug, which targets the energy metabolism of mycobacteria. Adenosine triphosphate (ATP) is an essential molecule for survival of all cells including mycobacteria. Mycobacteria persisting in well-encapsulated lung cavities and in endosomes of macrophages can survive even under low oxygen molecular tension leading to resistance against standard TB drugs. ${ }^{4}$ Nevertheless, ATP production by ATP synthase is essential regardless the nature of mycobacteria like active or dormant, replicating or non-replicating, extracellular or intracellular and fermenting or non-fermenting. ${ }^{5}$

ATP synthase complex, the site of ATP production, is located in inner membrane of mycobacterial mitochondria. It is composed of two structural domains; membrane embedded $\mathrm{F}_{0}$ domain and cytoplasmic located $\mathrm{F}_{1}$ domain with three subunits (a, b2 \& c10-15) and five subunits $(\alpha 3, \beta 3, \gamma, \delta \& \varepsilon)$ respectively. The $F_{0}$ domain with 10-15 ' $c$ ' subunits are arranged in the form of discs and the $\beta$ subunit of $F_{1}$ domain has catalytic activity which combines one ADP with Pi to form ATP. Proton motive force generated across mitochondrial membrane by electron transfer chain, fuels the rotation of discs and results in activation of catalytic $\beta$ subunit of $F_{1}$ domain resulting in ATP generation. ${ }^{6-8}$

Protonated form of bedaquiline has high affinity for the ATP synthase complex. It specifically binds with a distinct position at the interface of ' $a$ ' and ' $c$ ' subunit in $\mathrm{F}_{0}$ domain and blocks rotation of discs and thereby the entire process of ATP synthesis. In vitro studies also proved that binding affinity of bedaquiline is not influenced by excess proton, $\mathrm{pH}$ or other electrostatic interactions. ${ }^{9}$ Human ATP synthase complex have similar domain configuration with very narrow protein sequence similarity. ${ }^{10}$ This information can assure that bedaquiline might be a safe drug in terms of pharmacodynamic aspects.

\section{Pharmacokinetics}

In study involving healthy volunteers with single dose of bedaquiline in doses of 10,30,100, 300 and $450 \mathrm{mg}$ and multiple daily doses ranging from 25 to $400 \mathrm{mg}$ for 14 days, the maximum plasma concentration $\left(C_{\max }\right)$ was attained after $5 \mathrm{~h}\left(T_{\max }\right)$. The attained $C_{\max }$ comparatively follows linear relationship with the dose administered, which was evident from variation of $C_{\max }$ from $0.07 \mu \mathrm{g} / \mathrm{ml}$ after a single dose of $10 \mathrm{mg}$ to $9 \mu \mathrm{g} / \mathrm{ml}$ for a dose of 700 mg. ${ }^{11}$ Bedaquiline undergoes an oxidative metabolism via CYP3A4 enzyme and produces an active but comparatively less efficacious N-desmethyl metabolite (M2), which further metabolized into M3 and M4 (N-demethylation). In vitro studies have proved that cellular phospholipidosis inducement is stronger in M2 than bedaquiline. This cellular phospholipidosis is responsible for development of adverse effects like QT prolongation, hepatotoxicity and myopathy. However the concentration of M2 and bedaquiline attained in in-vivo studies did not produce such adverse effects even at $C_{\text {max. }}{ }^{12}$

Bedaquiline follows triphasic elimination and characterized by an outstandingly long terminal half-life, around $173 \mathrm{~h}$ in humans. This is very important because it may allow intermittent drug administration when it is combined with others drugs in the regimen for MDR-TB and XDR-TB. ${ }^{13}$ Half-life of M2 is comparatively higher than bedaquiline, around $230 \mathrm{~h}$. However this has minimal significance as $\mathrm{M} 2$ is not as active as bedaquiline even at $\mathrm{C}_{\max }{ }^{14}$

\section{Preclinical and clinical studies}

In a study done in murine models of $\mathrm{TB}$, bedaquiline given at a dose of $25 \mathrm{mg} / \mathrm{kg}$ without any combination was as effective as WHO regimen for MDR-TB. Group of mice treated with bedaquiline $25 \mathrm{mg} / \mathrm{kg}$ along with $\mathrm{H}, \mathrm{R}$ and $\mathrm{Z}$ exhibited lung culture negativity for AFB immediately after six weeks of treatment. ${ }^{15}$ Combination of bedaquiline $2.5 \mathrm{mg} / \mathrm{kg}$ with $\mathrm{Z}$ compared with bedaquiline $25 \mathrm{mg} / \mathrm{kg}$ alone demonstrated similar results. As pyrazinamide disrupts the mycobacterial membrane potential and thereby reduces ATP synthesis, an effective synergism is ensued when bedaquiline and $Z$ are combined. This synergistic property is important in treating MDRTB because $Z$ is the most active drug against persistent mycobacteria and further augmented by adding RPT with bedaquiline and Z. ${ }^{16}$ Amongst all anti MDR-TB drugs bedaquiline $(25 \mathrm{mg} / \mathrm{kg}$ ) has displayed highest bactericidal potency and second best sterilizing activity. ${ }^{17}$ This is important because regimen aiming at decreasing the treatment duration should consider both the sterilizing activity and bactericidal potency. On contrary, bedaquiline is bacteriostatic against Mycobacterium avium indicating that same dosage schedule cannot be followed and dose increment is required in case of M. avium TB. $^{18}$

In simulating the exact pathogenesis of human TB, like development of granulomatous lymphadenitis, pulmonary and extrapulmonary $\mathrm{TB}$, guinea pig models are better than murine models. ${ }^{19}$ Two studies conducted in guinea pig TB models demonstrated bedaquiline when combined with $\mathrm{R}$ and $\mathrm{Z}$ causes rapid clearance of secondary lesions due to hematogenous spread. Bacterial loads in lungs, lymph nodes and spleen were dropped to undetectable level just after four weeks of treatment. ${ }^{20,21}$

All these studies with murine and guinea TB models have shown that bedaquiline has been non-toxic and could eradicate both TB as well as MDR-TB infection. Adding bedaquiline with other two traditional drugs cleared the infection faster than standard fourdrug regimen. Furthermore, combining bedaquiline with AMK, $\mathrm{ETH}, \mathrm{MOXF}$ and $\mathrm{Z}$ showed greater efficacy against MDR-TB. ${ }^{21}$

The single most important factor considered for testing the new anti-TB drug in human after evaluation of safety by preclinical studies is early bactericidal activity (EBA) of that drug. EBA reflects an ability of drug to reduce the bacterial load rapidly. An EBA study conducted in 75 patients has shown that bedaquiline at a dose of $400 \mathrm{mg}$ once daily displayed significant bactericidal activity with similar magnitude of $\mathrm{H}$ and $\mathrm{R}$. However no bactericidal action occurred during the first 2-4 days of treatment, which may be due to its unique mechanism of action. After blocking ATP synthesis, it takes at least three to four days from depletion of ATP to disruption of intracellular $\mathrm{pH}$ homeostasis finally culminating in bactericidal activity. $^{22}$

A randomized, phase II, placebo-controlled trial was conducted in 47 patients to evaluate the safety, efficacy and tolerability in two stages. Efficacy of bedaquiline is reflected as anti-mycobacterial activity, which is measured in terms of sputum negative conversion rate and decline in median log 10 count of colony forming units (CFUs). In stage 1 addition of bedaquiline to standard therapy for MDR-TB significantly reduced the time for sputum conversion, as compared with placebo $(P=0.003)$. The rate of conversion to negative culture was $48 \%$ in bedaquiline group (10/21) while it was $9 \%$ in placebo $(2 / 23)$. At the end of 8 weeks treatment period, the median log 10 CFU counts declined rapidly in bedaquiline than placebo and reduction exceeded placebo at all-time points. ${ }^{23}$

Same patients were followed for another 24 weeks in stage 2 of this study. Statistical analysis done in stage 2 was more stringent than stage 1 . This is because only the subjects with sputum conversion at the end of 24 weeks were taken into account and those who discontinued were left behind and carried forward as not 
converted. Extraordinarily efficacy of was maintained the same in stage 2 also which was reflected by significant difference between two groups for time to $50 \%$ culture conversion. It took 78 days for bedaquiline group while in placebo it took 129 days to attain $50 \%$ culture conversion $(P=0.031){ }^{24}$

In another randomized, placebo controlled, phase II clinical trial conducted in 161 MDR-TB patients, it was shown that bedaquiline group attained earlier sputum conversion rate. At the end of 24 weeks higher sputum conversion rate was noted in bedaquiline group (79\%) than placebo group (58\%). ${ }^{25}$ All these encouraging results from these clinical trials formed the strong basis for approval of bedaquiline for the treatment of MDR-TB by FDA.

\section{Safety}

Being 20,000 times more selective inhibitor for mycobacterial ATP synthase complex than human ATP synthase complex, bedaquiline could be predicted as a safe drug in terms of pharmacodynamic aspects. ${ }^{10}$ Both bedaquiline and M2 have a property of developing cellular phospholipidosis, which is responsible for QT interval prolongation, hepatotoxicity and neuropathy. In a study done in 75 patients by Rustomjee et al, administration of bedaquiline $400 \mathrm{mg}$ daily was associated with very few minor adverse events like hemoptysis (21.4\%), rash (7\%), diarrhea (7\%) and somnolence (7\%). Even though the classical phospholipidosis induced QT prolongation (around $10 \mathrm{~ms}$ ) was observed in all patients, it is insignificant and it was seen in all treatment groups. Moreover, pathologically prolonged QT or corrected QT values were also not observed in any treatment groups. ${ }^{22}$

In study done by Diacon et al for evaluation of safety, tolerability, and pharmacokinetics, few minor side effects like nausea, diarrhea, hemoptysis and hyperuricemia were reported. No deaths were reported in this study. ${ }^{24}$ However in another trial conducted in 161 MDR-TB patients 10 out of 79 (12.7\%) in bedaquiline group and 2 of $81(2.5 \%)$ in placebo group died. ${ }^{25}$ According to this trial data, death rate in bedaquiline groups was nearly five times higher than the placebo group with significant difference $(P=0.017)$. Five out of 12 patients died in bedaquiline group and all deaths in placebo group were due to nature of the disease. Nevertheless remaining deaths in bedaquiline group could not be explained clearly.

On taking all these facts into account, one can come to a conclusion that only minor, tolerable side effects were revealed in early phase of the study. Long-term monitoring should be done to bring out the potential life threatening adverse effects of bedaquiline. Recently WHO has advised the interim guidelines for usage of bedaquiline which focused mainly on safety. It laid five definite criteria to be met before administration of bedaquiline in MDR patients namely, proper inclusion criteria, proper informed consent, close monitoring facilities, strict adherence to WHO recommended regimen, foreseeing and avoiding drug interactions. ${ }^{26}$ Hence for complete understanding about the clinical potential of bedaquiline, more data on safety and tolerability are required.

\section{Resistance}

Based on minimum inhibitory concentration (MIC), bedaquiline shows excellent bactericidal activity against $M$. $t b$, Mycobacterium avium, Mycobacterium intracellulare and Mycobacterium leprae. Its spectrum of activity also covers both rapid and slow growers among non-tuberculous mycobacteria (NTM). Amongst rapid growing NTM, Mycobacterium chelonae, Mycobacterium fortuitum, Mycobacterium phlei and Mycobacterium vaccae are susceptible as their MIC is less than $0.5 \mathrm{ug} / \mathrm{ml}$. Mycobacterium kansasii, Mycobacterium gordonae, Mycobacterium simiae and other strains belonging to slow growing NTM also displayed susceptibility for bedaquiline.
However a particular strain of $M$. tb and two mutant strains in Mycobacterium smegmatis have developed resistance against bedaquiline. ${ }^{27}$ This acquired resistance is due to two important point mutations occurring in atpE gene, which encodes for subunit 'c' protein. ${ }^{28}$ In mutant subunit ' $c$ ' protein, the amino acid aspartate at 28 th position and alanine in 63 rd position is replaced by proline and valine respectively. The resultant shorter carbon chain due to this substitution will prevent bedaquiline from interfering with passage of proton ions and hence ATP synthesis cannot be blocked. $^{29}$

Interestingly some species like Mycobacterium xenopi, Mycobacterium novo-castrense and Mycobacterium shimoidei have natural resistance against bedaquiline because the usually well preserved alanine amino acid in 63rd position in subunit ' $c$ ' is replaced by methionine residue in these bacteria. ${ }^{27,30}$ This shows that blocking ATP production may not result in bactericidal activity in all mycobacteria.

Acquisition of resistance to bedaquiline is inversely related to plasma concentration and duration of treatment. However in clinical trials, subjects achieved sputum conversion rapidly which resulted in lower bacterial sputum load preventing development of resistance. ${ }^{24}$ In spite of this assurance, development of resistance for bedaquiline forecasts great caution while designing regimen for MDR-TB and XDR-TB.

\section{Drug interactions}

A drug having fewer interactions with other anti-TB drugs and anti-HIV drugs is one of the most important properties expected during development of newer TB drugs. Bedaquiline dissatisfied this norm partially because it has drug interaction with efavirenz and rifampicin though of less significance.

Even though no human studies were performed till now to study interactions between bedaquiline and rifampicin, murine models have shown that bedaquiline sustains its high EBA even at half dose when administered along with rifampicin. This proves that even if there is significant interaction leading to decreased bedaquiline level when given along with rifampicin, it might not be significant clinically. ${ }^{15}$

Bedaquiline, being a CYP3A4 substrate and CYP3A4 is induced by efavirenz; co-administration leads to decreased AUC of bedaquiline from $58155 \mathrm{ngh} / \mathrm{ml}$ to $52135 \mathrm{ngh} / \mathrm{ml}$. Efavirenz also increases the level of bedaquiline metabolite M2. However, in terms of development of adverse effects, there was no significant difference in results obtained between bedaquiline alone and combination group. Decrease in bedaquiline concentration also was insignificant clinically. ${ }^{12}$ On contrary, AUC of bedaquiline is increased by $22 \%$ when co-administered with lopinavir and ritonavir and hence bedaquiline should be used judiciously in this situation only when benefit outweighs the risk. However, no dosage change is required when bedaquiline is given along with nevirapine. ${ }^{11}$

Since bedaquiline cannot be administered as a monotherapy ever, it should be always given in combination with other anti-MDR TB drugs. Hence more human studies regarding interactions of bedaquiline with other anti-MDR TB drugs are mandatory. Only after acquiring detailed information about these drug interaction studies, the best and effective anti-MDR TB regimen can be formulated.

\section{Conclusion}

Emergence of MDR-TB, XDR-TB and TDR-TB demands a better tussle against control of tuberculosis. Although newer targets, innovative drugs and effective combination regimen against $\mathrm{TB}$ bacilli are in obligatory need, no anti-TB drug has been approved by 
drug regulatory authorities for the past three decades except bedaquiline. Bedaquiline, a mycobacterial ATP synthase inhibitor, exhibited excellent efficacy in preclinical and clinical trials having bactericidal and sterilizing activity against $M$. tb and many NTM. It successfully decreased the duration for sputum conversion period and median CFUs when compared with placebo in patients with MDR-TB. However an increased death rate, occurrence of serious side effects and trivial drug interactions with rifampicin and efavirenz warrants the caution for its usage. Moreover development of resistance in some mycobacterial strains naturally as well as acquired indicates that not all mycobacteria are susceptible to bedaquiline. Overall, bedaquiline could deliver the best in terms of efficacy but requires more studies regarding safety.

\section{Conflicts of interest}

All authors have none to declare.

\section{References}

1. WHO Global Tuberculosis Report. WHO; 2013 [Internet]. [cited 2013 Nov 27] Available from: http://www.who.int/tb/publications/global_report/en/.

2. WHO Treatment of Tuberculosis: Guidelines for National Programmes [Internet]. WHO. [cited 2013 Nov 27]. Available from: http://www.who.int/tb/ publications/tb_treatmentguidelines/en/.

3. Spigelman M, Woosley R, Gheuens J. New initiative speeds tuberculosis drug development: novel drug regimens become possible in years, not decades. Int J Tuberc Lung Dis Off J Int Union Tuberc Lung Dis. 2010;14:663-664.

4. Berney M, Cook GM. Unique flexibility in energy metabolism allows mycobacteria to combat starvation and hypoxia. PLoS One. 2010;5:e8614.

5. Tran SL, Cook GM. The F1Fo-ATP synthase of Mycobacterium smegmatis is essential for growth. J Bacteriol. 2005;187:5023-5028.

6. Hirono-Hara Y, Noji H, Nishiura M, et al. Pause and rotation of F(1)-ATPase during catalysis. Proc Natl Acad Sci U S A. 2001;98:13649-13654.

7. Yasuda R, Masaike T, Adachi K, Noji H, Itoh H, Kinosita Jr K. The ATP-waiting conformation of rotating F1-ATPase revealed by single-pair fluorescence resonance energy transfer. Proc Natl Acad Sci U S A. 2003;100:9314-9318.

8. Kasho VN, Boyer PD. Vacuolar ATPases, like F1,F0-ATPases, show a strong dependence of the reaction velocity on the binding of more than one ATP per enzyme. Proc Natl Acad Sci U S A. 1989;86:8708-8711.

9. Haagsma AC, Podasca I, Koul A, et al. Probing the interaction of the diarylquinoline TMC207 with its target mycobacterial ATP synthase. PLoS One. 2011;6:e23575.

10. Haagsma AC, Abdillahi-Ibrahim R, Wagner MJ, et al. Selectivity of TMC207 towards mycobacterial ATP synthase compared with that towards the eukaryotic homologue. Antimicrob Agents Chemother. 2009;53:1290-1292.

11. Drugs@FDA: FDA Approved Drug Products [Internet]. [cited 2013 Nov 27]. Available from: http://www.accessdata.fda.gov/scripts/cder/drugsatfda/index. $\mathrm{cfm}$ ? fuseaction $=$ Search. DrugDetails.

12. Dooley KE, Park J-G, Swindells S, et al. Safety, tolerability, and pharmacokinetic interactions of the antituberculous agent TMC207 (bedaquiline) with efavirenz in healthy volunteers: AIDS Clinical Trials Group Study A5267. J Acquir Immune Defic Syndr. 2012:59:455-462.

13. Andries K, Verhasselt P, Guillemont J, et al. A diarylquinoline drug active on the ATP synthase of Mycobacterium tuberculosis. Science. 2005;307:223-227.

14. Rouan M-C, Lounis N, Gevers T, et al. Pharmacokinetics and pharmacodynamics of TMC207 and its N-desmethyl metabolite in a murine model of tuberculosis. Antimicrob Agents Chemother. 2012;56:1444-1451.

15. Lounis N, Gevers T, Van Den Berg J, Andries K. Impact of the interaction of R207910 with rifampin on the treatment of tuberculosis studied in the mouse model. Antimicrob Agents Chemother. 2008;52:3568-3572.

16. Ibrahim M, Andries K, Lounis N, et al. Synergistic activity of R207910 combined with pyrazinamide against murine tuberculosis. Antimicrob Agents Chemother. 2007;51:1011-1015.

17. Andries K, Gevers T, Lounis N. Bactericidal potencies of new regimens are not predictive of their sterilizing potencies in a murine model of tuberculosis. Antimicrob Agents Chemother. 2010:54:4540-4544.

18. Lounis N, Gevers T, Van den Berg J, Vranckx L, Andries K. ATP synthase inhibition of Mycobacterium avium is not bactericidal. Antimicrob Agents Chemother. 2009;53:4927-4929.

19. Basaraba RJ, Dailey DD, McFarland CT, et al. Lymphadenitis as a major element of disease in the guinea pig model of tuberculosis. Tuberc Edinb Scotl. 2006;86: 386-394.

20. Shang S, Shanley CA, Caraway ML, et al. Activities of TMC207, rifampin, and pyrazinamide against Mycobacterium tuberculosis infection in guinea pigs. Antimicrob Agents Chemother. 2011;55:124-131.

21. Lenaerts AJ, Hoff D, Aly S, et al. Location of persisting mycobacteria in a Guinea pig model of tuberculosis revealed by r207910. Antimicrob Agents Chemother. 2007;51:3338-3345.

22. Rustomjee R, Diacon AH, Allen J, et al. Early bactericidal activity and pharmacokinetics of the diarylquinoline TMC207 in treatment of pulmonary tuberculosis. Antimicrob Agents Chemother. 2008;52:2831-2835.

23. Diacon AH, Pym A, Grobusch M, et al. The diarylquinoline TMC207 for multidrug-resistant tuberculosis. N Engl J Med. 2009;360:2397-2405.

24. Diacon AH, Donald PR, Pym A, et al. Randomized pilot trial of eight weeks of bedaquiline (TMC207) treatment for multidrug-resistant tuberculosis: longterm outcome, tolerability, and effect on emergence of drug resistance. Antimicrob Agents Chemother. 2012;56:3271-3276.

25. Use of Bedaquiline (TMC-207) for treatment of MDR-TB [Internet] Int Union Against Tuberc Lung Dis; 2012 [cited 2013 Nov 27]. Available from: http:// uwclh.conference2web.com/content/2168.

26. WHO Interim Guidance on the Use of Bedaquiline to Treat MDR-TB [Internet]. WHO. [cited 2013 Nov 27]. Available from: http://www.who.int/tb/challenges/ mdr/bedaquiline/en/index.html.

27. Huitric E, Verhasselt P, Andries K, Hoffner SE. In vitro antimycobacterial spectrum of a diarylquinoline ATP synthase inhibitor. Antimicrob Agents Chemother. 2007;51:4202-4204.

28. Koul A, Dendouga N, Vergauwen K, et al. Diarylquinolines target subunit c of mycobacterial ATP synthase. Nat Chem Biol. 2007:3:323-324.

29. De Jonge MR, Koymans LHM, Guillemont JEG, Koul A, Andries K. A computational model of the inhibition of Mycobacterium tuberculosis ATPase by a new drug candidate R207910. Proteins. 2007;67:971-980.

30. Segala E, Sougakoff W, Nevejans-Chauffour A, Jarlier V, Petrella S. New mutations in the mycobacterial ATP synthase: new insights into the binding of the diarylquinoline TMC207 to the ATP synthase C-ring structure. Antimicrob Agents Chemother. 2012;56:2326-2334. 